\title{
INFORMES
}

\section{INVESTIGACIÓN NECESARIA EN MATERIA DE POBLACIÓN}

\section{INTRODUCCIÓN}

1. El examen de las prioridades de la investigación demográfica ha de tener en cuenta las finalidades a que deban servir los resultados de tal investigación. Es muy posible que los intereses y prioridades del experto teórico en demografía que estudia las interrelaciones entre diferentes variables demográficas o entre éstas, por una parte, y las variables sociales y económicas, por otra, difieran de los del administrador especialmente interesado en los problemas del desarrollo o en los programas de población, por lo que cada uno de ellos puede aplicar criterios diferentes para definir "lo necesario". Normalmente, el administrador buscará resultados de aplicación inmediata. Sin embargo, la investigación que tiene por objeto sentar las bases de los programas de acción deberá tener en cuenta los conocimientos existentes sobre interrelaciones demográficas y deberá basarse sólidamente en las generalizaciones teóricas empíricas de que ya se dispone para explicar los cambios demográficos. Dicha investigación no sería posible a menos de existir ya cierto conocimiento básico de los factores que influyen en el crecimiento de las poblaciones humanas. Son ya bien conocidos los vínculos precisos existentes entre las variables demográficas básicas, tales como la fecundidad, la mortalidad y la estructura por edad. Pero en demografía, al igual que en otras ramas del saber, una mayor investigación fundamental puede conducir a un mejor entendimiento de los problemas prácticos. Por consiguiente, a falta de los sistemas adecuados de confección de censos y de estadísticas del registro civil con que se tropieza en muchas regiones del mundo, se han ideado métodos que permitan, partiendo de los datos incompletos que se obtienen, llegar a conclusiones acerca del estado de desarrollo demográfico. Los supuestos en que se basan estos métodos son algo restrictivos y por ello es necesario estudiar sus posibilidades de aplicación a aquellas situaciones en las que no intervienen necesariamente. A primera vista, esta clase de investigación, que supone el empleo de modelos de población y de métodos de simulación, quizá parezca totalmente teórica pero aun así puede aportar mejores resultados que algunos estudios que de momento parezcan encerrar mayor importancia práctica. Estos problemas se examinan más adelante en el párrafo 9.

2. En el presente documento se intentará evaluar la necesidad de una investigación demográfica que permita obtener una visión más clara del panorama demográfico actual, mejorar los métodos de efectuar pronósticos y sugerir medios que influyan en el crecimiento de la población. No se

* Documento E/CONF. 60/CBP/28, presentado a la Conferencia Mundial de Población de Bucarest, Rumania, preparado por el personal de la Unión Internacional para el Estudio Científico de la Población. Las opiniones son las de los autores y no necesariamente las de las Naciones Unidas. 
examinarán las disposiciones que podrían adoptarse para establecer procedimientos tendientes a lograr una mejor información demográfica. Todavía hay muchos países en los que se han levantado muy pocos censos, o en los que no se ha levantado ninguno, y cuya calidad de datos demográficos básicos es deficiente. Ni qué decir tiene que conviene contar con una información demográfica básica mejor y más adecuada sobre aquellas regiones para las cuales se carece actualmente de ella, asunto al que debe asignarse una alta prioridad. Ello supone perfeccionar el levantamiento de censos y la reunión de estadísticas demográficas, mediante una colaboración entre estadísticos y demógrafos. En varios países y en los últimos años se ha tropezado con cierta oposición a que se levantaran censos y se obtuvieran estadísticas demográficas por temor a inmiscuirse en la vida privada de los ciudadanos. Esto ha creado dificultades para administrar esos servicios. Importa comprender las razones que han provocado semejante actitud y adoptar medidas para contrarrestar sus consecuencias. Pero no se considera que esto constituya "investigación" en el sentido dado a esta palabra en el presente documento.

3. También se han excluido del presente trabajo las investigaciones que puedan precisarse en materia biomédica, tales como las relativas al perfeccionamiento de técnicas anticonceptivas o la utilización de métodos abortivos mejores o más seguros. De estos temas se ocupa el documento preparado para la Conferencia por la Organización Mundial de la Salud (OMS). ${ }^{1}$ Sin embargo, algunos de los temas que se examinan más adelante versan sobre el campo de la biología.

4. Cabe mencionar otra cuestión preliminar. Existe actualmente un déficit considerable de especialistas capacitados en investigación demográfica. Esto significa que han tenido que abandonarse varios trabajos convenientes de investigación o que ha habido que encomendarlos a personas cuya formación fundamental corresponde a otras disciplinas. Incluso se ha dicho que ni siquiera se han podido aprovechar adecuadamente los datos de que ya se disponía en algunos censos de determinados países por falta de personal capacitado para analizar, evaluar y comprender a fondo la información presentada en las estadísticas oficiales. Como ejemplo de ello se ha citado la serie de censos de 1960 en la región de la Comisión Económica para Asia y el Lejano Oriente (CEPALO). Así pues, todo plan de ampliación de la investigación demográfica debe presuponer un aumento del número de personas capacitadas en esa esfera y por tanto también de los medios de formación. Tales medios deben ponerse a disposición de las personas que empiezan a trabajar; en particular, es importante que los recién graduados reciban formación en organizaciones que normalmente sólo ofrecen empleo a quienes ya poseen varios años de experiencia práctica.

5. El examen de las necesidades y prioridades puede abordarse de varias maneras. Las subdivisiones tradicionales de la demografia, tales como la mortalidad, la nupcialidad, la fecundidad, las migraciones, etc., podrían considerarse por separado. También podría establecerse una distinción entre la investigación necesaria en los países desarrollados y la que se requiere en los países en desarrollo, ya que las necesidades de ambos difieren. En el presente documento se utilizan ambas maneras de abordar la cuestión. Tras un breve estudio del estado actual de la investigación demográfica, se exa-

1 Véase el documento E/CONF. 60/CBP/22. 
minan las necesidades concretas de los países en desarrollo, en los que los demógrafos se ven obligados a adoptar técnicas especiales de observación $\mathrm{y}$ análisis. A continuación se examina la fecundidad, tanto en las regiones desarrolladas del mundo como en aquellas que están en vías de desarrollo, incluyendo en este tema la nupcialidad y la investigación en materia de planificación de la familia. También se examinan la mortalidad y los problemas generales de salud. Por último, dentro del título general "Relaciones entre Población y Desarrollo Económico y Social", se estudian las migraciones, la urbanización, los problemas generales del medio, la estructura de la población, la política de población y los problemas de "predicción".

\section{BREVE ESTUdio DEL ESTAdo ACTUAL DE LA INVESTIGACión}

6. En los últimos diez años se ha registrado un aumento espectacular del interés general que despiertan las cuestiones demográficas. A ello han contribuido los dos factores siguientes:

a) el aumento de la población, principalmente en los países subdesarrollados, pero últimamente en los países en desarrollo;

b) la necesidad de proteger el medio y el peligro de agotar algunos recursos naturales.

Por ello se ha dado prioridad a los estudios sobre la fecundidad (especialmente sobre la planificación de la familia) y el desarrollo económico, y a la investigación fundamental se han preferido los estudios cuyos resultados puedan tener aplicación inmediata.

7. El rápido aumento de los trabajos que tuvo efecto en esas esferas sólo fue posible gracias al esfuerzo conjunto de varias organizaciones, que también desempeñaron una función importante al coordinar la investigación y la difusión de sus resultados. Entre ellas merecen especial mención las Naciones Unidas, que en 1966 establecieron el Fondo de las Naciones Unidas para Actividades en Materia de Población (resolución 2211 (XXI) de la Asamblea General) y declararon el año 1974 como Año Mundial de la Población (resolución 2683 (XXV) de la Asamblea General), su División de Población, las comisiones económicas regionales y los organismos especializados de las Naciones Unidas (Organización Mundial de la Salud, Organización Internacional del Trabajo, Organización de las Naciones Unidas para la Agricultura y la Alimentación, Organización de las Naciones Unidas para la Educación, la Ciencia y la Cultura), los institutos regionales de formación profesional en materia de población y la Organización de Cooperación y Desarrollo Económicos, así como organizaciones tales como la Unión Internacional para el Estudio Científico de la Población, el Estudio Mundial sobre la Fecundidad, varias fundaciones internacionales públicas y privadas de Asia, América y Europa, la Federación Internacional de Planificación de la Familia y muchas universidades de todo el mundo.

8. Se han dejado un poco al margen algunas esferas de investigación. Por ello se estima que en los años venideros la investigación demográfica no debe proseguirse al mismo ritmo en todas sus ramas, sino que es preciso establecer de nuevo las prioridades, como se intenta hacerlo en el presente documento, para llenar las lagunas que existen en la actualidad. 


\section{INFORMACIÓN NECESARIA EN LOS PAÍSES EN DESARROLLO}

9. Ya se ha mencionado la baja calidad de la información demográfica de que actualmente se dispone en muchas regiones del mundo. En el Sudán y el Zaire desde mediados del decenio $1950-1960$ no se ha levantado ningún censo ni se ha llevado a cabo ningún muestreo importante. En Etiopía nunca se ha levantado ningún censo completo, si bien se ha deducido cierta información demográfica a partir de datos por muestreo. No es posible poner remedio inmediato a esta situación, ya que se tardará en elaborar un sistema adecuado de empadronamiento y estadísticas demográficas. La falta de datos fidedignos hace que sea especialmente difícil estimar las estructuras de mortalidad postinfantil. A su vez, dificulta la estimación de la estructura por edad y sexo de esas poblaciones. Se han aprovechado las informaciones sobre orfandad para estudiar la mortalidad de adultos y se han elaborado otros métodos de estimación indirecta. La mayoría de esas nuevas técnicas se basan en el supuesto de que las tasas de los sucesos vitales han permanecido constantes a lo largo del tiempo. Es preciso analizar la solidez de estos procedimientos frente a las variaciones que tienen efecto en la hipótesis de índices vitales constantes. Tal análisis puede requerir la simulación mediante computadoras de diferentes poblaciones modelo que tengan antecedentes demográficos variables. A primera vista estos estudios parecerían tener escasa utilidad práctica pero, dado el tiempo que inevitablemente se precisa para mejorar los sistemas de levantamiento de censos y de administración de las estadísticas demográficas, así como la urgente necesidad de contar con mejor información, la investigación encaminada a mejorar la calidad de los métodos de estimación parece merecer alta prioridad.

10. También es necesario que se lleve a cabo una mayor labor experimental con respecto a la precisión con que se registran mediante diversos métodos los sucesos vitales corrientes, el envío de recordatorios y el carácter de las encuestas de control o de calidad. En esta labor se incluirían estudios sobre los métodos de muestreo, la preparación de cuestionarios y los errores en las encuestas. Estos últimos pueden ser especialmente importantes y su estudio está relativamente descuidado. Así, muchos de los cuestionarios empleados en encuestas demográficas están redactados en un idioma europeo pero se presentan a los censados en lengua vernácula y a menudo la traducción corre a cargo del agente censal. Es preciso evaluar en qué medida la precisión de la respuesta se ve afectada por este procedimiento; por ejemplo, es más importante que la traducción sea más que literal, una expresión clara del significado de la pregunta. Muchos de estos problemas son de carácter estadístico y administrativo y no corresponden a la demografía propiamente dicha. Sin embargo, su estudio es de importancia capital para el progreso de los conocimientos demográficos. Actualmente no se publica toda esta información. Convendría disponer de evaluaciones sistemáticas y de un sistema de comparabilidad internacional, por lo que la labor del Estudio Mundial sobre la Fecundidad ${ }^{2}$ puede contribuir a arrojar luz sobre esta cuestión.

2 Programa internacional de investigación sobre la fecundidad realizado por el Instituto Internacional de Estadísticas, de Voorburg (Países Bajos), con la colaboración de las Naciones Unidas y en cooperación con la Unión Internacional para el Estudio Científico de la Población, de Lieja (Bélgica). 
11. En los últimos años se han ensayado dos métodos de investigación en países subdesarrollados: la confrontación de datos procedentes de dos fuentes independientes y las encuestas realizadas en varias fases. Ha habido cierto desacuerdo sobre las ventajas e inconvenientes relativos de cada uno de estos dos métodos. Convendría realizar estudios de costos-beneficios de uno $\mathbf{y}$ otro e investigar en qué circunstancias cada método resulta especialmente adecuado.

\section{MORTALIDAD}

12. Ya se ha hecho referencia a la falta de información sobre las estructuras de mortalidad, especialmente por lo que respecta a los paises en desarrollo. La situación es especialmente grave en el África tropical, donde pudiera decirse que las estructuras de la mortalidad de adultos constituye una incógnita demográfica. La situación es apenas mejor en algunas otras regiones en desarrollo. Poco se sabe acerca de las tasas de mortalidad por edades y la información sobre las causas de muerte es casi inexistente. Será preciso obtener esa información mediante encuestas especiales por muestreo. Deberían realizarse estudios sobre la diferencia de mortalidad entre los sexos, especialmente en aquellas zonas en que la mortalidad femenina parece ser superior a la masculina, con el fin de identificar los factores que originan una inversión de la díferencia usual entre ambos sexos. A este respecto, debe prestarse especial atención a la influencia de la mortalidad derivada de la maternidad y de las consecuencias de múltiples partos, así como a las diferencias de nutrición.

13. En el párrafo 9 ya se ha mencionado la necesidad de mejorar el método de estimación; además, sería muy conveniente elaborar una nueva serie de tablas de mortalidad modelo para sustituir las confeccionadas hace unos 15 años por las Naciones Unidas. ${ }^{3}$ Estas tablas, que han sido útiles como un primer paso, eran principalmente de un solo parámetro. La elaboración de tablas modelo más perfeccionadas aplicables a los países en desarrollo sería muy útil para el estudio de la mortalidad. Ya se ha hecho algo en esta esfera, especialmente elaborando tablas regionales y de parámetros múltiples. Pero todas ellas se basan en la muestra de las tablas de vida real empleadas en la elaboración del modelo, muestra que no abarca todas las regiones del globo ni todas las posibles estructuras de mortalidad.

14. También es importante observar las variaciones de las tasas de mortalidad de los países en desarrollo en el transcurso del tiempo. Entre otras cosas, dichas tasas se emplean para proyecciones demográficas y en aquellas regiones del mundo en que la mortalidad todavía es elevada. Las variaciones de los supuestos relativos a la mortalidad futura pueden influir de manera importante en las estimaciones de la población futura. A este respecto, un detenido estudio de la experiencia de las regiones desarrolladas en las que la disminución de la mortalidad a determinadas edades parece haberse detenido puede constituir un importante indicador que permita conocer mejor la situación en las regiones menos desarrolladas.

15. Frecuentemente se estudia la mortalidad no sólo por el interés pro-

3 Modelos de mortalidad por sexo y edad; tablas modelo de mortalidad para países insuficientemente desarrollados, publicación de las Naciones Unidas, N: de venta: 1955-XIII-9. 
pio del tema sino también porque constituye un indicador del nivel de salud de que disfruta una población. Sin embargo todavía no se conoce suficientemente la relación, existente entre las condiciones sanitarias y las tasas de mortalidad; por ejemplo, aún deben estudiarse más a fondo el efecto de la erradicación del paludismo, la influencia de los niveles de nutrición sobre la mortalidad, el efecto de las variaciones de la dimensión de la familia y del orden de los nacimientos en las posibilidades de muerte. También merece mayor atención el evidente cese de la disminución de la mortalidad en los hombres de edad madura.

16. En general, los conocimientos sobre la mortalidad son mucho mayores en los países desarrollados, ya que el estudio de sus tasas fue uno de los primeros aspectos estudiados de la demografía. Sin embargo, todavía existen lagunas, especialmente con respecto a la mortalidad en ambos extremos de la escala de la vida. En muchas regiones desarrolladas han persistido las diferencias sociales en la mortalidad infantil y perinatal (e incluso en la mortalidad de adultos), aunque haya disminuido el nivel absoluto de las tasas. Es preciso proseguir la investigación para tratar de identificar las condiciones que van unidas a las tasas de mortalidad superiores al promedio.

17. Es probable que en las regiones desarrolladas el número de personas muy ancianas (de 80 y más años de edad) siga aumentando en lo que resta del siglo. Ello dará lugar a mayores necesidades de servicios sociales y médicos para este sector de la población. Las tasas de mortalidad de las personas muy ancianas han reaccionado en menor medida a los cambios que experimentan las condiciones sociales y económicas que las tasas de mortalidad de períodos menos avanzados de la vida. Incluso en los países que poseen buenos sistemas de estadísticas demográficas es frecuente que las tasas de mortalidad de las personas muy ancianas hayan de obtenerse por estimación. Será preciso prestar mayor atención de la que se ha prestado hasta ahora a los cambios inherentes a la edad, a la estructura de las causas, a la longevidad extrema y al problema de la "supervivencia por medicación".

18. En muchos países se han publicado estadísticas de las causas de defunción, pero éstas no siempre se han analizado a fondo. Sería conveniente estudiar la interdependencia de las diferentes causas y elaborar una tipología de las estructuras de mortalidad, que sería útil para realizar estudios de mortalidad comparativa.

\section{FECUNDIDAD}

19. Uno de los objetivos importantes de la investigación demográfica es mejorar los métodos de proyección y predicción de la población. Para conseguirlo, es de importancia fundamental seguir estudiando la fertilidad y la fecundidad, tanto en los países desarrollados como en los países en desarrollo, lo que permitiría obtener resultados más inmediatos que con estudios sobre mortalidad. En las regiones desarrolladas ésta ha disminuido hasta llegar a un nivel muy bajo y las variaciones en los supuestos sobre la mortalidad futura probablemente sólo afecten a la pobación de edad más avanzada; en las regiones en desarrollo, aunque la mortalidad es mayor, la fecundidad es con mucho el factor más importante que puede influir en los movimientos futuros de población. 
20. En el aspecto de la medición, la fertilidad, más que la fecundidad, es el sector en el que se ha adelantado menos. Hasta ahora se ha hecho relativamente poco en cuanto a las diferencias de la capacidad biológica de concepcción y reproducción, tanto entre diferentes poblaciones como entre diferentes estratos de la misma población. No se sabe si existen diferencias étnicas de fertilidad. Para colmar esta laguna deberia estudiarse la fecundidad natural de las diferentes poblaciones y esos estudios también constituirían un requisito previo para evaluar la eficacia de distintos programas anticonceptivos.

21. Sin embargo, en los países desarrollados los estudios sobre la capacidad biológica de concepción y reproducción son menos importantes que los relativos a las tasas de reproducción real. En esta esfera la investigación puede hacerse a nivel macro y microsocial. En el primer caso, es preciso conocer con más claridad las variables sociales y económicas que se hallan asociadas a las diferencias de fecundidad. A este respecto, son importantes los estudios comparativos y del nivel cultural y cabe esperar que el material reunido en el Estudio Mundial sobre la Fecundidad aporte nuevos conocimientos. Entre los factores que deben tenerse en cuenta figuran la diferencia de nivel de educación, los índices de desarrollo económico, los cambios que experimenta la condición social de la mujer y los diversos tipos de estructura de parentesco y de familia. Todos estos factores tendrían que estudiarse en diferentes fases de desarrollo. Esta labor se ha iniciado ya en buena parte y las publicaciones especializadas en demografía se hallan repletas de monografías que dan cuenta del resultado de diferentes investigaciones. Sin embargo, apenas se han coordinado los esfuerzos llevados a cabo, por lo que no siempre cabe comparar entre sí los resultados de esos estudios. Tal vez convendría que existiera una colaboración más estrecha entre las diferentes organizaciones nacionales de investigación.

22. También en este terreno, algunos estudios que a primera vista parecen ser meramente académicos pueden contribuir a un mejor conocimiento del crecimiento transitorio. Por ejemplo, los detallados estudios históricos recientemente realizados de países europeos en los que en el contexto del conjunto nacional se han examinado con detenimiento las estadísticas de pequeñas zonás. De modo más general, cabe considerar la historia de la población como un laboratorio en el que se han realizado múltiples experimentos que pueden analizarse con provecho. La observación de la marcha evolutiva actual (más fácil de realizar y en la que concurren casos mejor conocidos) encierra mayor interés y podría mejorar mucho mediante una cooperación internacional más estrecha. Tampoco debería descuidarse el estudio de determinadas poblaciones o subpoblaciones que presentan estructuras o comportamiento de fecundidad anómalos.

23. Los estudios a nivel macrosocial comprenderán también trabajos sobre la asociación existente entre urbanización y fecundidad. Históricamente, el éxodo a las ciudades en los países desarrollados ha conducido a una menor fecundidad en estas zonas y a la aparición de diferenciales entre ellas y el campo. En los países en desarrollo el proceso de urbanización no siempre ha dado lugar a semejantes diferenciales, por lo que importa estudiar la evolución de la fecundidad urbana dentro del mundo en desarrollo.

24. En los estudios macrosociales se ha venido descuidando en cierto modo la cuestión de la nupcialidad. La dinámica de la nupcialidad sigue conociéndose sólo imperfectamente y merecería la pena estudiar los factores 
que influyen en la formación y disolución no sólo de los matrimonios legales sino de las uniones sexuales en general. En especial, se requiere estudiar la relación entre las variables económicas y la nupcialidad en diferentes países y culturas y la relación entre nupcialidad y fecundidad.

25. La labor a nivel macrosocial puede arrojar luz sobre las asociaciones empíricas existentes entre los fenómenos demográficos por una parte y las variables socioeconómicas por otra, pero la mayor parte de la tarea pendiente corresponde al nivel microsocial. La fecundidad nacional es el resultado de un conjunto de muchas decisiones individuales y sólo mediante un proceso de desagregación y un estudio de parejas individuales cabe comprender y apreciar adecuadamente las diferencias de fecundidad entre los países y dentro de un mismo país. Además de los estudios comparativos, deberá darse cada vez más importancia a estudios longitudinales que permitan investigar con detalle los acontecimientos demográficos que se producen en el ciclo vital de los individuos y evaluar la influencia de los factores "perturbadores", tales como los cambios de empleo, las migraciones y el desempleo. Asimismo, esos estudios permitirán relacionar los cambios de actitud con las variaciones del comportamiento demográfico. Tales estudios, si se planean y realizan cuidadosamente, pueden proporcionar una información sumamente útil, incluso a base de muestras pequeñas, de unos pocos miles de individuos. De hecho, la necesidad de llevar una administración cuidadosa constituye un argumento en favor de que la muestra sea pequeña.

26. Es preciso conseguir información acerca del proceso real de decisión en materia de fecundidad. Hasta ahora la mayoría de los estudios que se han realizado en esta esfera se han centrado en la actitud de la mujer con respecto a la formación de la familia y la fecundidad. Relativamente pocos son los estudios efectuados sobre la actitud del hombre. No obstante, incluso en los países desarrollados donde la planificación de la familia está tan ampliamente extendida y la emancipación de la mujer es mayor, cabe albergar dudas respecto a que sea la mujer, y no la pareja conjuntamente, quien adopte todas las decisiones en materia de fecundidad. Donde la mujer todavía ocupa un lugar subordinado, la actitud y las motivaciones del hombre pueden tener más peso que las de la mujer y este sector, que ha estado relativamente descuidado, debe ser objeto de mayor atención.

27. También requiere mayor estudio el carácter de las decisiones en materia de dimensión de la familia. Hasta ahora muchos estudios se han dedicado a investigar la dimensión preferida y esperada del total de la familia. Pero es casi seguro que la actitud relativa a la fecundidad varía en el curso de formar una familia, por lo que parece preferible estudiar esa actitud en diferentes etapas de la formación, es decir, saber si una pareja con $n$ hijos vivos debe volver a tener un hijo del orden $n+1$. A este respecto deberá tenerse en cuenta la distribución por sexo de las familias en esé mómentó. En esta esfera puede ser útil la colaboración entre demógrafos y antropólogos sociales, sobre todo si han de realizarse estudios culturales. Se confía asimismo en que el Estudio Mundial sobre la Fecundidad realice trabajos en esta esfera.

28. Los estudios acerca del proceso de decisión individual a nivel microsocial, que exigen mucha organización, incluso la contratación de un equipo de entrevistadores capacitados, son de difícil realización. Estos estudios tropiezan también con las incertidumbres' y dificultades de toda encuesta relacionada con actitudes. Además, no es posible uniformar a escala 
mundial ni su formato ni los cuestionarios, ya que deben tenerse en cuenta las diferentes circunstancias sociales y económicas de las diferentes regiones. Aunque cierto grado de uniformación y coordinación sea conveniente, esto no es lo que más importa; constituyen el único medio de entender mejor determinados conceptos (número ideal de hijos, embarazos no deseados) en las diferentes culturas y de descubrir nuevos indicadores del comportamiento relativo a la fecundidad.

29. Debe tenerse en cuenta la estructura general de las relaciones dentro de cada familia y la influencia aue reina sobre las decisiones en materia de fecundidad. Ulteriores estudios sobre la sociología de la familia permitirían conocer mejor el papel que desempeñan sus diferentes miembros, su participación en el progreso decisorio y la importancia de la familia en la transmisión de generación en generación de los valores de fecundidad en las diferentes sociedades.

\section{Planificación de la familia y evaluación de los programas DE PLANIFICACIÓN DE LA FAMILIA}

30. En el párrafo 19 se ha mencionado ya la conveniencia de mejorar el estudio de la fertilidad. Se requieren mejores métodos para evaluar la eficiencia de los diferentes tipos de contracepción que tienen por objeto evitar los nacimientos que tendrían efecto sin el uso de medios anticonceptivos. También es preciso investigar más a fondo el concepto "nacimientos impedidos".

31. Conviene proseguir los estudios sobre contracepción denominados CAP (conocimiento-actitud-práctica). Debe tratarse de conseguir una mayor comparabilidad de las encuestas. Hay que realizar estudios para determinar los mejores métodos de difundir información anticonceptiva y de inducir a las parejas a que utilicen material anticonceptivo y los servicios que se ponen a su disposición. Estos estudios deben asociarse a la labor sobre el proceso decisorio individual y familiar descrito en los párrafos 27 a 29 .

32. Conviene proseguir los estudios para establecer cómo las políticas oficiales pueden influir en la tasa de variación de la población. Necesariamente, estos estudios deberán vincularse a las investigaciones sobre motivaciones individuales ya mencionadas. Sin embargo, la labor deberá ampliarse a los problemas administrativos que supone la aplicación de políticas de población y las probables repercusiones de tales medidas sobre la política económica general y los servicios sociales; por ejemplo, la liberalización de las leyes sobre el aborto y la disponibilidad de servicios ginecológicos generales. Si se fomentara la reducción de la fecundidad mediante medidas fiscales habría que encontrar la manera de desalentar la fecundidad sin perjudicar a los hijos ya nacidos. No hay que dejar de tener presentes las consecuencias sociales y distributivas de las medidas fiscales que se adopten por razones demográficas (sean pronatalistas o antinatalistas).

\section{ReLACIONES GENERALES ENTRE POBLACióN Y DESARROLlO ECONÓMICO Y SOCIAL}

33. El comienzo del crecimiento económico (especialmente cuando va ligado al desarrollo industrial) normalmente lleva consigo una modificación 
de la distribución geográfica de la población. Los habitantes se trasladan a nuevos centros de atracción (cuando se fundan nuevas industrias o nuevas ciudades) o, más a menudo, a ciudades ya existentes. En los municipios urbanos, la migración interna e internacional es un factor determinante del crecimiento de la población mucho más importante que el crecimiento natural. Constituye un factor importante de urbanización. Es menester mejorar las técnicas de análisis de este proceso mediante una definición más precisa de las aglomeraciones urbanas, la subdivisión de estas áreas en zonas más homogéneas, el estudio de las áreas de alta concentración y la observación minuciosa del crecimiento de las nuevas ciudades. Los estudios históricos del crecimiento urbano constituyen asimismo un medio de aumentar nuestros conocimientos.

34. Es preciso estudiar mucho más a fondo los motivos y las condiciones de la migración a las grandes ciudades y otros lugares. Las razones que impulsan a abandonar el lugar de origen, la elección del momento de la emigración (especialmente en relación con el ciclo de la familia), la elección del lugar de destino, el papel que desempeña la vivienda y los efectos de las medidas de planificación física sólo pueden comprenderse mediante un análisis minucioso de los datos disponibles (obtenidos por enumeraciones censales y otros medios) y mediante encuestas especiales. Debe estudiarse asimismo la integración de los migrantes en su lugar de destino y las consecuencias para el lugar de origen. Tampoco deben descuidarse las migraciones estacionales ni el traslado de los trabajadores al lugar de trabajo; esto último, en especial, acarrea costos sociales y tiene repercusiones importantes en el tiempo disponible de los individuos.

35. El párrafo anterior se refería principalmente a la migración interna, pero también es aplicable en su mayor parte a la migración internacional. No obstante, la migración internacional plantea otros problemas específicos. En primer lugar está el problema de su medida. Los datos son frecuentemente más escasos y menos exactos que los relativos a la migración interna; a menudo faltan datos sobre los inmigrantes y los relativos a los emigrantes son casi inexistentes. El demógrafo se encuentra ante un problema análogo al que plantea la estimación del crecimiento natural de la población en un país que carece de un sistema de estadísticas del estado civil. Se precisa una metodología especial, y, hasta ahora, es muy poco lo que se ha hecho en este sentido. Los efectos de la migración en la estructura por edad de la población de los paises de inmigración y emigración no son insignificantes. Además, es menester prestar mayor atención a los efectos de la migración sobre las tasas de natalidad de los países de salida y de entrada. Es preciso examinar los problemas de asimilación e integración de los inmigrantes en los paises de entrada, especialmente cuando se dan grandes diferencias culturales entre los inmigrantes y la población indígena. Es necesario recoger datos sobre el éxito de las medidas adoptadas para evitar la discriminación y disminuir el riesgo de conflicto entre distintos grupos inmigrantes y entre inmigrantes indígenas. Por lo que respecta a la migración temporal, se sabe muy poco acerca de la duración de la permanencia de los migrantes temporales, las condiciones de la migración de retorno y las consecuencias de las migraciones temporales en los países de origen de los migrantes.

36. Recientemente los economistas han prestado más atención que antes al factor demográfico en el desarrollo económico. Sin embargo, es me- 
nester utilizar medidas demográficas más refinadas para el estudio del desarrollo económico y aumentar el número de parámetros demográficos en el modelo. Deberían tenerse en cuenta las consecuencias económicas de un crecimiento más lento de la población y, en los países desarrollados, las posibles consecuencias de una disminución del número de habitantes o de las fluctuaciones del volumen de población. Estos hechos afectan a la estructura de la población y, aunque sus efectos quizás tarden en dejarse sentir, son difícilmente reversibles. Una variación súbita de la tasa de natalidad puede tener consecuencias más inmediatas e importantes; varios países han experimentado tales variaciones en los últimos años. De un modo más general, la rapidez con que se alcanza un objetivo demográfico determinado puede dar lugar a perturbaciones de la estructura de la población que quizás sean duraderas.

37. El economista y el demógrafo están interesados en el estudio de 1a fuerza de trabajo; éste suele hacer hincapié en la influencia de las estructuras demográficas y su desarrollo. Es importante hacerse cargo de que la mano de obra no es un factor de producción homogéneo y de que no es fácil sustituir a un hombre por otro ni transferirlos de un lugar a otro sin crear problemas. En otras palabras: se necesita un sistema de contabilidad de la mano de obra (en el que se distingan los diferentes niveles de calificación y capacitación) para suplir los recuentos meramente financieros. Es evidente que los problemas de formación y capacitación no pueden ser considerados exclusivamente en términos financieros. Será menester prestar una atención especial a los problemas de la educación femenina y de las mujeres que ejercen una actividad lucrativa y sus relaciones con la fecundidad y la composición de la población activa.

38. Durante los últimos años se ha registrado un súbito interés por los estudios referentes a la relación entre los recursos de población y el medio ambiente, estudios que se han multiplicado. Tales estudios han tenido demasiado a menudo un carácter global y en ellos las variables demográficas han sido tratadas de manera algo simplista. Es necesario profundizar mucho más para comprender las relaciones utilizadas en estos modelos; por ejemplo, las relaciones entre la mortalidad y la contaminación y deterioración del medio ambiente, las consecuencias del hambre y la malnutrición en la fecundidad y la mortalidad, la influencia de una densidad de población muy alta en el stress, las tensiones de la vida diaria, etc. Debería alentarse la investigación en estos campos.

39. Las relaciones recíprocas entre población, recursos y medio ambiente sólo pueden estudiarse bajo una perspectiva a largo plazo. Hay otro aspecto de los estudios de población que también requiere una perspectiva a largo plazo, es decir, la genética de poblaciones. Esta disciplina, que a primera vista parece ser abstracta y teórica, es aplicable al estudio de los sistemas matrimoniales, el control de la fecundidad, la morbilidad y la migración. Por consiguiente, es conveniente apoyar las investigaciones en este campo y fomentar la colaboración entre demógrafos y genetistas.

40. Debe mencionarse también la necesidad de estudiar los efectos de las políticas de población. El primer problema que se plantea en esta esfera es el de la determinación de los objetivos. En el caso de la fecundidad, a menudo se establece explícitamente que las medidas de política están encaminadas a influir en un sentido determinado en los movimientos de la fecundidad. Se ha mencionado ya la dificultad de evaluar los efectos de los 
programas de planificación de la familia (párrafo 32). Sin embargo, las políticas de población pueden abarcar un ámbito mucho más amplio: mortalidad y morbilidad (sanidad pública), migración interior (planificación física), migración exterior (controles fronterizos), nupcialidad (política familiar), etcétera. Sería útil simplemente enumerar las áreas en que se ha tratado de influir en las variables demográficas, directa o indirectamente, por medios voluntarios o coactivamente. Los estudios retrospectivos y las observaciones de los actuales movimientos de la población pueden reemplazar los experimentos, cuya realización es imposible en las ciencias sociales. Es pues, especialmente importante observar atentamente la experiencia de aquellas regiones en desarrollo en las que recientemente se haya reducido la tasa de crecimiento y estudiar las circunstancias que han conducido a tal disminución.

41. Finalmente, para que los demógrafos puedan contribuir a evaluar los efectos de las decisiones políticas y a vaticinar sus consecuencias, hay que mejorar ante todo la exactitud de la predicción demográfica. Se utiliza deliberadamente el término "predicción" para significar el empleo de técnicas muy concretas de extrapolación de las tendencias con miras a estimar lo más exactamente posible los valores futuros. La investigación debe tender a determinar las características principales de las modificaciones que con el tiempo se operen en las variables demográficas pertinentes y a establecer una teoría verificable de los componentes y determinantes del movimiento de la población. La apreciación de las propuestas de investigación y la determinación del orden de prioridades deberían llevarse a cabo con arreglo a este criterio. 